\title{
Light-matter interactions at the nanoscale: challenges and opportunities
}

\author{
Kenneth B. Crozier \\ School of Physics and Department of Electrical and Electronic Engineering \\ University of Melbourne \\ Victoria 3010, Australia \\ kcrozier@unimelb.edu.au
}

\begin{abstract}
Optical methods are challenging to apply at the nanoscale, due to the large mismatch between the wavelength and the sizes of nanoscale objects. I will describe projects that have addressed this challenge for various applications.
\end{abstract}

Keywords-photodetectors, nanostructure fabrication, subwavelength structure, optical resonator

\section{SEMICONDUCTOR NANOWIRE PHOTODETECTORS FOR MULTISPECTRAL IMAGING}

We present recent studies in which engineering the interaction between light and nanoscale materials has been pursued for applications in image sensors and in spectroscopy. In the first, we present recent work by the author and colleagues on the use of silicon [1-5] and germanium [6] nanowires for multispectral imaging. We show that their ability to support waveguide modes leads to spectrally-selective absorption properties [1]. We show that this in turn enables the nanowires to be used as filters or photodetectors for color and multispectral imaging [2-6].

\section{OPTICAL TRAPPING WITH PLASMONIC AND PHOTONIC NANOSTRUCTURES}

A second theme of this presentation concerns optical nanotweezers.

The development of integrated approaches for optical trapping, based on photonic or plasmonic structures fabricated on a chip, offers several compelling advantages. First, chip-based optical traps enable the trapping platform to be miniaturized. Second, the chipbased configuration lends itself naturally to the incorporation of sensing modalities. Third, optical nanostructures can generate strong near-fields, boosting the trapping performance.
In this presentation, works by the author and his team in the field of optical trapping with silicon photonics and with plasmonics are described. We have pursued various approaches to optical trapping. A selection of these works will be presented at the conference, based on the available time.

We have demonstrated the use of silicon microring resonators for trapping [7] and sensing [8] particles. We have furthermore demonstrated silicon photonics for sorting particles [9], as well as for sensing proteins [10]. We have performed experiments in which a silicon photonic crystal cavity trapped a silver nanoparticle on whose surface molecules had been formed. We carried out Raman spectroscopy of these molecules, with the silver nanoparticle held in position via the photonic crystal cavity [11]

Plasmonic nanostructures are compelling for optical trapping due to the large gradient forces they can generate, a consequence of their ability to generate highly confined optical fields. Yet deleterious thermal effects can also occur. We have demonstrated the use of a plasmonic nanotweezer with an integrated heat-sink [12]. In our recent work, fluorescence microscopy was used to track the position of a nanoparticle trapped by a double nanohole aperture [13].

\section{REFERENCES}

[1] K. Seo, M. Wober, P. Steinvurzel, E. Schonbrun, Y. Dan, T. Ellenbogen, and K. B Crozier, "Multicolored vertical silicon nanowires," Nano Letters 11, 1851 (2011)

[2] H. Park and K. B Crozier, "Multispectral imaging with vertical silicon nanowires," Scientific Reports 3, 2460 (2013)

[3] H. Park, Y. Dan, K. Seo, Y. J. Yu, P. K. Duane, M. Wober, K. B. Crozier, "Filter-free image sensor pixels comprising silicon nanowires with selective color absorption," Nano Letters 14, 1804 (2014) 
[4] H. Park and K. B Crozier, "Elliptical silicon nanowire photodetectors for polarization-resolved imaging," Optics Express 23, 7209 (2015)

[5] H. Park and K. B Crozier, "Vertically stacked photodetector devices containing silicon nanowires with engineered absorption spectra," ACS Photonics 2, 544 (2015)

[6] A. Solanki and K. B Crozier, "Vertical germanium nanowires as spectrally-selective absorbers across the visible-to-infrared," Appl. Phys. Lett. 105, 191115 (2014)

[7] S. Lin, E. Schonbrun, and K. Crozier, "Optical Manipulation with Planar Silicon Microring Resonators," Nano Letters 10, 2408 (2010)

[8] S. Lin and K.B. Crozier, "Planar silicon microrings as wavelengthmultiplexed optical traps for storing and sensing particles," Lab on a Chip 11, 4047-4051 (2011);
[9] S. Lin and K.B. Crozier, "An integrated microparticle sorting system based on near-field optical forces and a structural perturbation," Optics Express 20, 3367-3374 (2012);

[10] S. Lin and K.B. Crozier, "Trapping-Assisted Sensing of Particles and Proteins using On-Chip Optical Microcavities," ACS Nano 7, 1725 (2013)

[11] S. Lin, W. Zhu, Y. Jin and K.B. Crozier, "Surface Enhanced Raman Scattering with $\mathrm{Ag}$ Nanoparticles Optically Trapped by a Photonic Crystal Cavity," Nano Letters 13, 559 (2013)

[12] K. Wang, E. Schonbrun, P. Steinvurzel and K.B. Crozier, "Trapping and rotating nanoparticles using a plasmonic nano-tweezer with an integrated heat sink," Nature Communications vol. 2, 469 (2011)

[13] Z. Xu, W. Song and K.B. Crozier, "Optical tracking of a nanoparticle trapped by a double nanohole aperture", CLEO 2016 conference 


\section{University Library}

\section{- M M N E R VA A gateway to Melbourne's research publications}

Minerva Access is the Institutional Repository of The University of Melbourne

Author/s:

Crozier, KB

Title:

Light-matter interactions at the nanoscale: challenges and opportunities

Date:

2016-01-01

Citation:

Crozier, K. B. (2016). Light-matter interactions at the nanoscale: challenges and opportunities. [Abstract]. 2016 INTERNATIONAL CONFERENCE ON OPTICAL MEMS AND NANOPHOTONICS (OMN), 2016-September, https://doi.org/10.1109/OMN.2016.7565833.

Persistent Link:

http://hdl.handle.net/11343/294900 